\title{
Transport properties of alkanes through ceramic thin zeolite MFI membranes
}

\author{
Z.A.E.P. Vroon *, K. Keizer, M.J. Gilde, H. Verweij, A.J. Burggraaf \\ Laboratory for Inorganic Materials Science, Faculty of Chemical Technology, University of Twente, P.O. Box 217, 7500 AE Enschede, \\ Netherlands
}

Accepted 3 April 1995

\begin{abstract}
Polycrystalline randomly oriented defect free zeolite layers on porous $\alpha-\mathrm{Al}_{2} \mathrm{O}_{3}$ supports are prepared with a thickness of less than $5 \mu \mathrm{m}$ by in situ crystallisation of silicalite- 1 . The flux of alkanes is a function of the sorption and intracrystalline diffusion. In mixtures of strongly and weakly adsorbing gases and at high loadings of the strongly adsorbing molecule in the zeolite pore, the flux of the weakly adsorbing molecule is suppressed by the sorption and the mobility of the strongly adsorbing molecule resulting in pore-blocking effects. The separation of these mixtures is mainly based on the sorption and completely different from the permselectivity. At low loadings of the strongly adsorbing molecules the separation is based on the sorption and the diffusion and is the same as the permselectivity. Separation factors for the isomers of butane ( $n$-butane/isobutane) and hexane (hexane/2,2-dimethylbutane) are respectively high $(10)$ and very high $(>2000)$ at $200^{\circ} \mathrm{C}$. These high separation factors are a strong evidence that the membrane shows selectivity by size-exclusion and that transport in pores larger than the zeolite MFI pores (possible defects, etc) can be neglected.
\end{abstract}

Keywords: Ceramic membranes; Zeolite membranes; Gas separation; Gas permeation; ZSM-5/silicalite-1

\section{Introduction}

In the last decade a large number of papers were published on the design and application of ceramic membranes $[1,2]$. New techniques like sol-gel and chemical vapour deposition are developed for the preparation of ceramic membranes. A new preparation technique has recently been developed for the preparation of zeolite MFI membranes [3-7]. These zeolite membranes on porous ceramic and metal supports were prepared with various thickness $(5-100 \mu \mathrm{m})$ by in situ crystallisation. The pore-size of zeolite MFI (ZSM-5 and silicalite-1) is about $0.55 \mathrm{~nm}$ (see Fig. 1 [8]). The

\footnotetext{
* Corresponding Author.
}

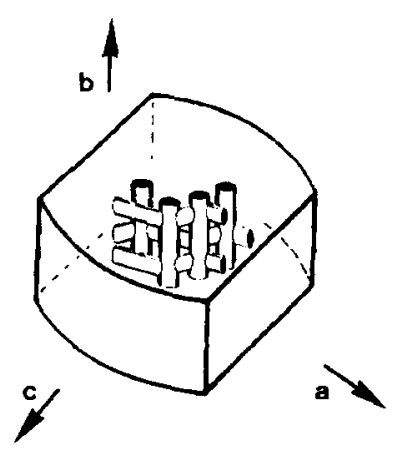

Fig. 1. Pore-structure of zeolite MFI | 101. 
kinetic diameters of linear alkanes and mono-branched alkanes are smaller than the pore-size of zeolite MFI and the kinetic diameters of di-branched alkanes are larger [9]. In "defect-free" zeolite MFI membranes molecular sieving is expected for di-branched alkanes (for example 2,2-dimethylbutane) and high separation factors are expected for mixtures of linear/di-branched alkanes (for example hexane/2,2-dimethylbutane mixture).

Studies of the transport properties of zeolite type membranes were initially performed by time-lag measurements of silicalite- 1 single crystals with a thickness of $100 \mu \mathrm{m}$ embedded in epoxy resin [10,11]. Diffusion constants of several gases, $\mathrm{C}_{1}-\mathrm{C}_{5}$, were obtained in this way. Results of these measurements are shown in Table 1. It is evident that the diffusion constant decreases with increase of the molecular length. The diffusion constants of the butane isomers are roughly the same. The first transport measurements on zeolite membranes prepared by in situ crystallisation showed that the separation performance of alkanes, $\mathrm{C}_{1}-\mathrm{C}_{4}$, strongly depends on process parameters.

This study reports the preparation of an MFI layer on an $\alpha-\mathrm{Al}_{2} \mathrm{O}_{3}$ support by in situ crystallisation. The $\alpha-\mathrm{Al}_{2} \mathrm{O}_{3}$ supported MFI layer has been characterized by single gas $\left(\mathrm{C}_{1}-\mathrm{C}_{4}\right)$ and multi-component (methane $/ n$-butane, $n$-butane/isobutane and hexane/2,2dimethylbutane) permeation measurements. In this paper the permeation results are discussed in a qualitatively way. A quantitative interpetation of the permeation results will be published elsewhere $[12,13]$

\section{Experimental}

\subsection{Membrane synthesis and characterization}

The preparation condition of membranes by the (multi) in situ crystallisation (MISC) method is presented schematically in Fig. 2. Zeolite films were grown in subsequent hydrothermal steps on an $\alpha-\mathrm{Al}_{2} \mathrm{O}_{3}$ support with a standard zeolite synthesis solution.

A home-made $\alpha-\mathrm{Al}_{2} \mathrm{O}_{3}$ disk (thickness $1.9-2.1 \mathrm{~mm}$, diameter $39 \mathrm{~mm}$, porosity $44-48 \%$ and pore-diameter 135-165 nm) was used as support for the membrane. The support was prepared by a process in which a commercial alumina powder (Philips, Uden) was pressed into a green compact at $100 \mathrm{MPa}$ by uni-axial
Table 1

Diffusion constants for hydrocarbons in zeolite single crystal by the membrane method

\begin{tabular}{ll}
\hline Gas & $D\left(\mathrm{~m}^{2} \mathrm{~s}^{-1}\right)$ \\
\hline Methane $^{\mathrm{a}}$ & $1 \times 10^{-10}$ \\
Ethane $^{\mathrm{a}}$ & $2 \times 10^{-11}$ \\
Propane $^{\mathrm{a}}$ & $7 \times 10^{-12}$ \\
$n$-Butane & $4 \times 10^{-12}$ \\
$n$-Butane $^{\mathrm{b}}$ & $1 \times 10^{-12}$ \\
& $3 \times 10^{-12}$ \\
Isobutane $^{\mathrm{b}}$ & $1 \times 10^{-12}$ \\
\hline
\end{tabular}

"Measured at $\left.60^{\circ} \mathrm{C} \mid 10\right\rceil$.

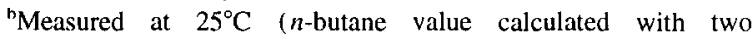
methods $) \mid 111$.

compaction with floating die. The green compact was sintered at $1230^{\circ} \mathrm{C}$ for $16 \mathrm{~h}$ (heating rate $100^{\circ} \mathrm{C} / \mathrm{h}$ ). The disk was polished on both sides. After polishing the supports were washed three times under ultra-sonic vibration for $10 \mathrm{~min}$. The supports were stored in water. A day before performing the hydrothermal treatment the supports were dried at $400^{\circ} \mathrm{C}$.

The standard zeolite synthesis solution was prepared by mixing sodiumhydroxide (Merck, >99\%) and tetrapropylammoniumhydroxide (Fluka, 20\% in water) at room temperature. After a clear solution was obtained silica (Baker, $99.75 \%$ ) was added to the solution. The silica was dissolved in the solution by stirring during heating. The molar ratio of the standard zeolite synthesis solution is $100 \quad \mathrm{SiO}_{2}: 15$ (TPA) ${ }_{2} \mathrm{O}: 5.3$ $\mathrm{Na}_{2} \mathrm{O}: 1420 \mathrm{H}_{2} \mathrm{O}$. After boiling for $300 \mathrm{~s}$ the solution was cooled down to room temperature in $1.5 \mathrm{~h}$. Water was added to compensate for the weight loss during the experiment [14].

The hydrothermal treatments were performed in a stainless steel autoclave (volume $3.43 \times 10^{-4} \mathrm{~m}^{3}$ ). The (dry) supports are positioned on the bottom of the (empty) autoclave and $\pm 100 \mathrm{~g}$ zeolite synthesis solution was carefully poured without hitting the supports. The autoclave was closed and heated under autogenous pressure at $120^{\circ} \mathrm{C}$ for 3 days. After the hydrothermal treatment the composite membrane was washed seven times with water. The temperature of the water used in the washing procedure was $60-85^{\circ} \mathrm{C}[14]$.

After the washing procedure the composite membrane was kept wet in water for $40 \mathrm{~min}$. The wet composite membrane is positioned on the bottom of the autoclave and $\pm 100 \mathrm{~g}$ aged standard zeolite syn- 


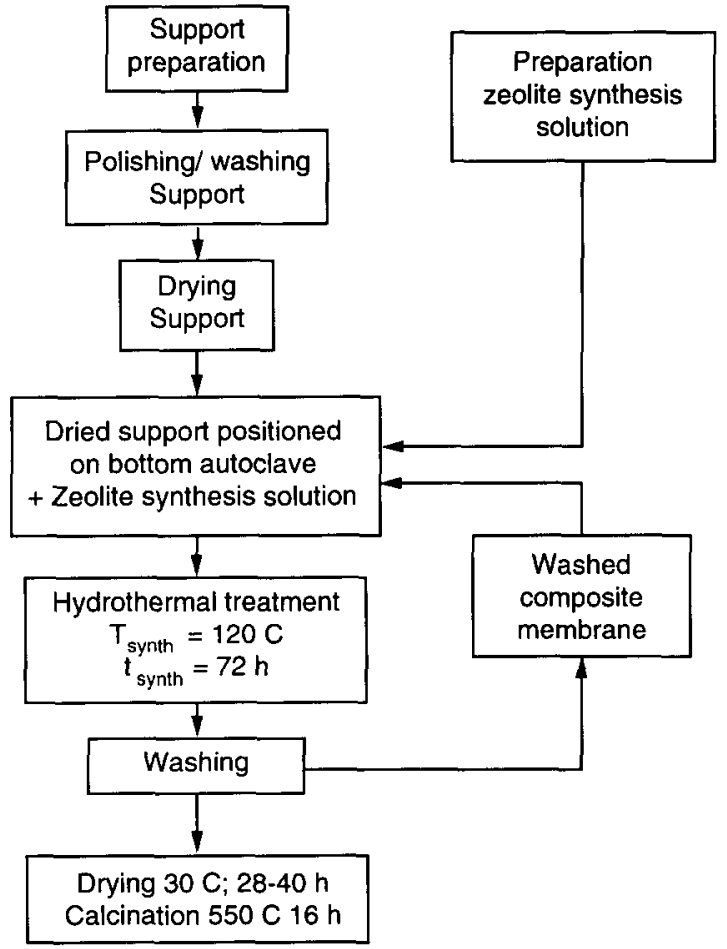

Fig. 2. Schematic representation of the preparation of zeolite MFI membranes by the multi in situ crystallisation (MISC) method.

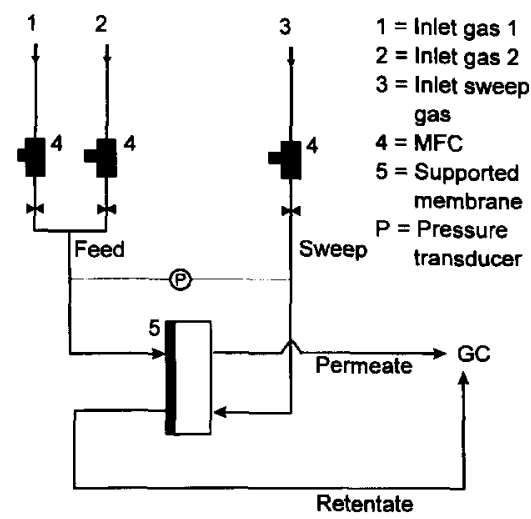

Fig. 3. Experimental configuration of Wicke-Kallenbach (counter current mode). MFC, mass flow Controller.

thesis solution was carefully poured without hitting the supports. The autoclave was again heated under autogenous pressure at $120^{\circ} \mathrm{C}$ for 3 days. After the hydrothermal treatment the composite membrane was again washed seven times.

After the last hydrothermal treatment the composite membranes were washed seven times. The composite membranes were dried in a laminar flow cupboard at $\pm 30^{\circ} \mathrm{C}$ for $16-40 \mathrm{~h}$. The template in the zeolite pores was removed at $550^{\circ} \mathrm{C}$ for $16 \mathrm{~h}$ (heating rate $10^{\circ} \mathrm{C} / \mathrm{h}$, cooling rate $20^{\circ} \mathrm{C} / \mathrm{h}$ ) [14].

The supported MFI layers were characterized by SEM, light microscopy and XRD.

\subsection{Transport measurements}

The membrane was placed in a stainless steel module (R-250, Velterop B.V.). O-rings (Kalrez, Dupont) were used as seals between the membrane and the module. The active (permeation) membrane area is 1.9 $\mathrm{cm}^{2}$.

Gas transport measurements were performed with a Wicke-Kallenbach set-up (counter current mode) in which no total pressure difference over the membrane is present (see Fig. 3). The gas flows and the composition of the feed were controlled by mass flow controllers (MFC, Brooks, type 5850 TR). Helium was used as sweep-and balance-gas. The total flow on both sides (feed and sweep) of the membranes was kept at $30 \mathrm{ml}$ (STP) $/ \mathrm{min}\left(20 \mu \mathrm{mol} \mathrm{s}^{-1}\right)$. A saturator was used in the measurements of hexane and 2,2-dimethylbutane. The purity of the used gases and liquids was always $>99.9 \%$. The composition of the feed, permeate and retentate were analyzed using a gas chromatograph (Varian 3400). The flux (for single gases and mixtures) and the separation factor (mixtures) were measured as a function of the (partial) feed pressure $\left(p_{\mathrm{f}}\right)$ and the temperature $(T)$. The separation factor $(\alpha)$ is defined as:

$\alpha=\frac{y}{1-y} \frac{1-x}{x}$

where $x$ and $y$ are, respectively, the molar fraction of gas $\mathrm{A}$ in the feed and permeate. The permselectivity $\left(P_{\alpha}\right)$ is defined as the ratio between single gas fluxes. The relative accuracy in the fluxes and calculated separation factors and permselectivities is in the order of $5 \%$.

\section{Results and discussion}

\subsection{Characterisation of the membrane}

In Fig. 4, SEM photographs of the cross-section (a) and the surface (b) of a zeolite MFI membrane are 

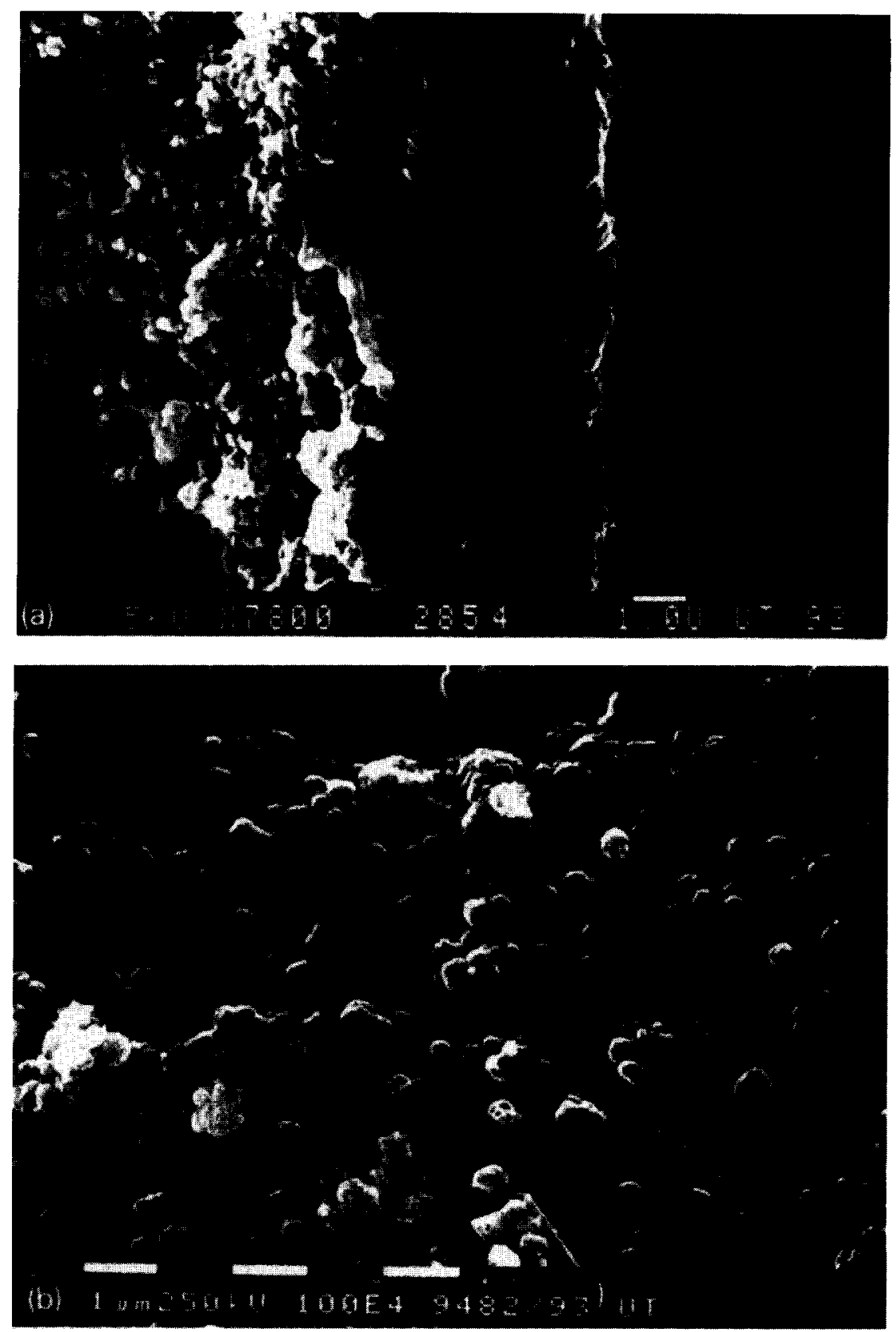

Fig. 4. Scanning electron micrographs of an $\alpha-\mathrm{Al}_{2} \mathrm{O}_{3}$ supported zeolite MFI membrane: (a) surface of the membrane; (b) cross section of the membrane.

shown. The zeolite membrane layer consists of a polycrystalline "dense" layer with a thickness smaller than $5 \mu \mathrm{m}$. The particles in the layer are randomly oriented and their size is $200-400 \mathrm{~nm}$.
In Fig. 5 the XRD pattern of a zeolite MFI membrane is given. The XRD pattern consists of the diffraction patterns of the support $\alpha-\mathrm{Al}_{2} \mathrm{O}_{3}$ and the zeolite MFI layer. The XRD pattern of MFI confirms the random 


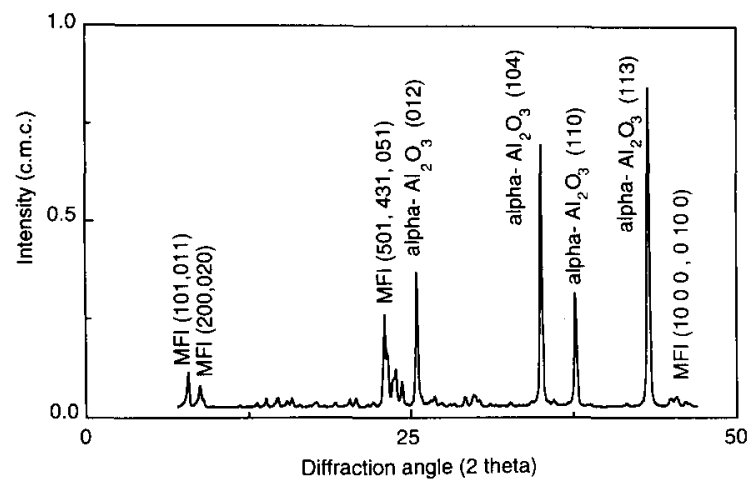

Fig. 5. X-ray diffraction pattern of an $\alpha-\mathrm{Al}_{2} \mathrm{O}_{3}$ supported zeolite MFI membrane.

orientation of the particles in the layer. No cracks were found by SEM and light microscopy.

The membranes were gas tight before calcination (permeation lower then $1 \times 10^{-12} \mathrm{~mol} \mathrm{~m}^{-2} \mathrm{~s}^{-1} \mathrm{~Pa}^{-1}$ ).

\subsection{Transport measurements}

In transient permeation measurements it is shown that the equilibrium times to reach steady state permeation are small $(<1 \mathrm{~h})$ for all mentioned gases, except for isobutane and 2,2-dimethylbutane below $100^{\circ} \mathrm{C}$ $[12,13]$.

In Fig. 6 the single gas flux of $n$-hydrocarbons is shown as a function of the partial feed pressure $(0-100$ $\mathrm{kPa}$ ) at 25 and $200^{\circ} \mathrm{C}$. At $200^{\circ} \mathrm{C}$ no large differences are present between the fluxes of the $n$-hydrocarbons and the fluxes increase almost linearly with partial feed pressure $(0-100 \mathrm{kPa})$. At $25^{\circ} \mathrm{C}$ the flux of ethane, propane and $n$-butane is not linear with the feed pressure. This non-linearity occurs at lower partial pressures for stronger adsorbing molecules.

In Fig. 7 the flux of the hydrocarbons is shown as a function of temperature at a feed pressure of $100 \mathrm{kPa}$. The flux as a function of temperature shows a maximum (except for methane). This maximum occurs at higher temperatures for stronger adsorbing molecules [16]. The maximum shifts towards lower temperatures at lower partial feed pressures, but the sequence in the maxima for different molecules remains the same. The differences in the fluxes of the gases are smaller than the differences in the diffusion constants presented in Table 1.

It can be concluded that the fluxes of the single gases are controlled both by the sorption of the hydrocarbons in the zeolite pores and the intracrystalline diffusion. A qualitative discussion will be given below, a quantitative treatment will be given elsewhere $[12,13]$. The maxima in the flux versus temperature can be explained by differences in sorption and diffusion of the molecules in the following way. The flux is proportional to the product of the concentration of the sorbed molecules in the zeolite pores and their diffusion constant. At low temperatures (or high pressures) and strongly adsorbing molecules the zeolite pores are almost completely filled with hydrocarbons. With increasing temperature the diffusion constant increases but the concentration change of sorbed molecules is of minor importance. This results in an increase of the flux

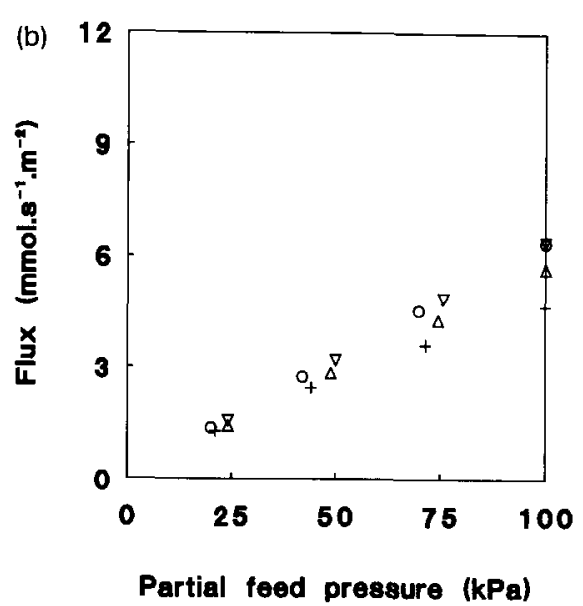

Fig. 6. Flux of methane $(O)$, ethane $(\nabla)$, propane $(\boldsymbol{\Pi})$ and $n$-butane $(+)$ as a function of partial feed pressure at $25(\mathrm{a})$ and $200^{\circ} \mathrm{C}(\mathrm{b})$. 


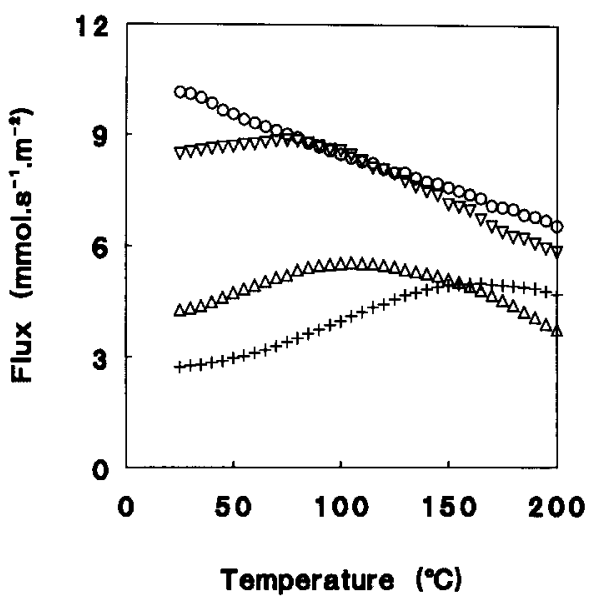

Fig. 7. Flux of methane $(O)$, ethane $(\nabla)$, propane $(\Delta)$ and $n$-butane $(+)$ as a function of temperature at a feed pressure of $100 \mathrm{kPa}$.

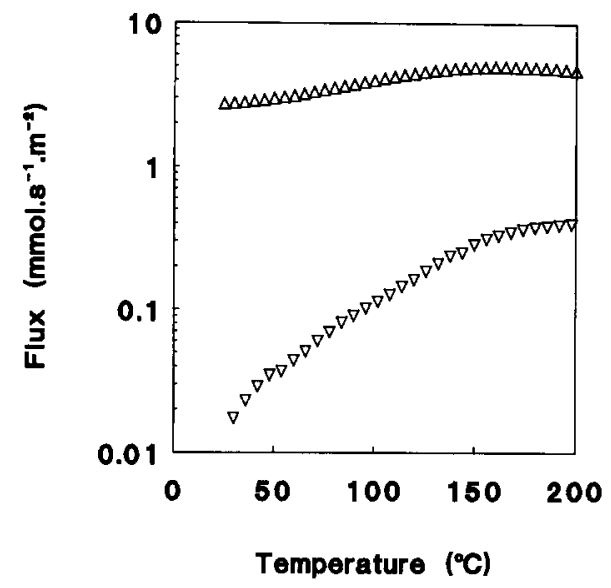

Fig. 8. Flux of $n$-butane $(\triangle)$ and isobutane $(\nabla)$ as a function of temperature at a feed pressure of $100 \mathrm{kPa}$.

through the membrane. At a certain temperature the decrease in the concentration of sorbed molecules supersedes the increase of diffusion and the total flux starts to decrease. The temperature at which the maximum flux occurs, depends on the partial feed pressure and type of molecule (sorption and diffusion). The sorption of the $n$-hydrocarbons in the zeolite pores increases with increasing $\mathrm{C}$-number and therefore the maximum in the flux versus temperature is at higher temperature for molecules with a higher $\mathrm{C}$-number.

The sorption is not the only factor that controls the flux as a function of the temperature. In Fig. 8 fluxes of $n$-butane and isobutane are shown. The $n$-butane flux has a maximum at $165^{\circ} \mathrm{C}$, the isobutane flux increases with increasing temperature. The adsorption capacity of isobutane is about $50 \%$ smaller than the adsorption capacity of $n$-butane [15]. If the sorption should be the only factor in controlling the flux, the maximum in the flux versus temperature of isobutane should be at a lower temperature than $n$-butane. This is not the case. Isobutane has a higher activation energy for diffusion than $n$-butane, so with increasing temperature the increase of the diffusion for isobutane is larger than for $n$-butane. This larger increase shifts the flux maximum temperature towards a higher temperature, because the decrease in the concentration of the sorbed isobutane molecules in the zeolite pores overrules the increase of diffusion at a higher temperature. The single gas flux of isobutane is almost two orders of magnitude lower than the single gas flux of $n$-butane at $25^{\circ} \mathrm{C}$. This value of the permselectivity is much larger than literature values for differences in sorption [15] and intercrystalline diffusion (see Table 1 ) for $n$-butane and isobutane.

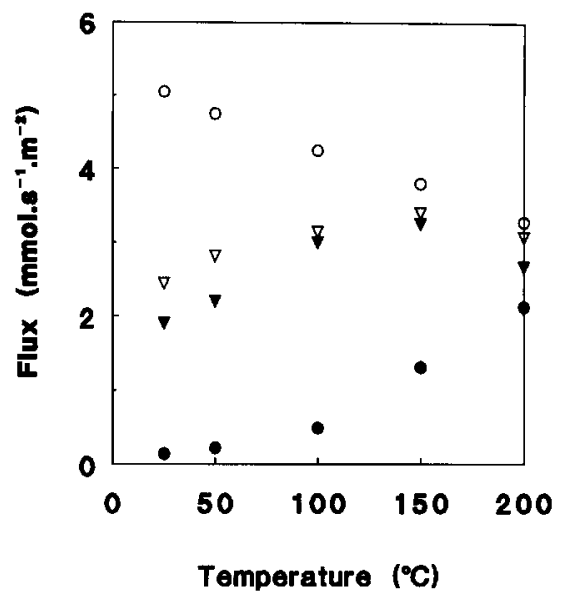

Fig. 9. Flux of $50 \mathrm{kPa}$ methane (o) and $50 \mathrm{kPa} n$-butane $(\nabla)$ as a function of temperature: $(O, \nabla)$ single gas; $(\boldsymbol{O}, \nabla)$ mixture.

Table 2

Comparison of the permselectivities and separation factors of $50 / 50$ $n$-butane (nb) /methane (me) and 50/50 n-butane/isobutane at 25 , 100 and $200^{\circ} \mathrm{C}$

\begin{tabular}{rllll}
\hline$T\left({ }^{\circ} \mathrm{C}\right)$ & $P_{r, \mathrm{me} / \mathrm{nb}}$ & $\alpha_{\mathrm{me} / \mathrm{nb}}$ & $P_{\alpha, \mathrm{nb} / \mathrm{ib}}$ & $\alpha_{\mathrm{nb} / \mathrm{ib}}$ \\
\hline 25 & 2.7 & 0.06 & 90 & 52 \\
100 & 1.4 & 0.16 & 50 & 19 \\
200 & 1.2 & 0.70 & 11 & 11 \\
\hline
\end{tabular}



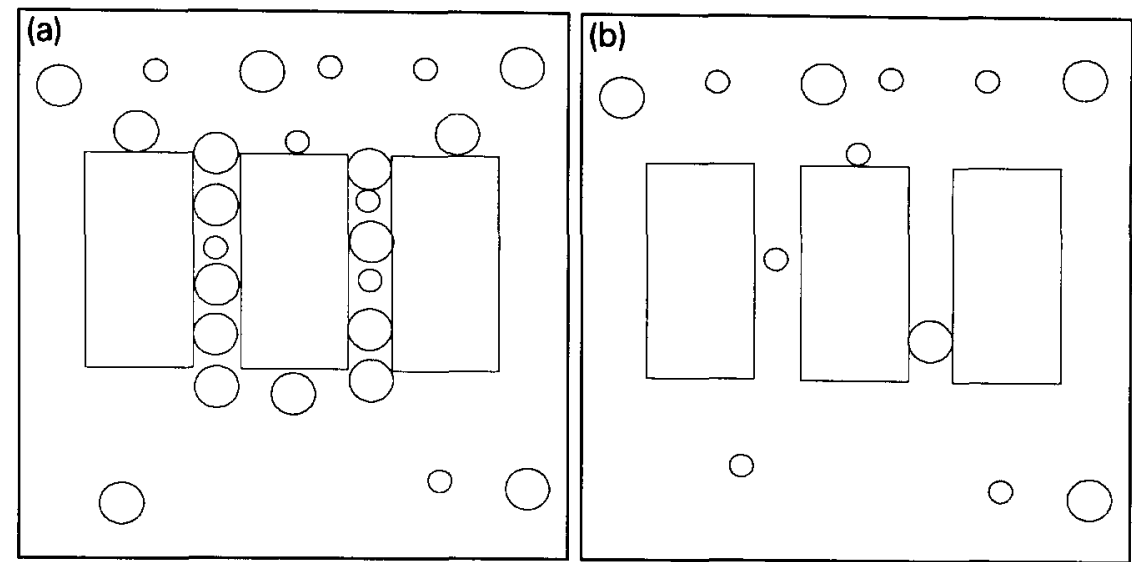

Fig. 10. Schematic presentation of transport of a mixture of strongly (large circles) adsorbing and weakly (small circles) adsorbing gas at high (a) and low (b) loading of molecules in the zeolite pores

Fig. 9 shows the large effect of $n$-butane sorption on the flux of methane as a function of the temperature. The presence of $n$-butane decreases the flux of methane at $25^{\circ} \mathrm{C}$ by a factor of 50 . The methane flux in the presence of $n$-butane increases with increasing temperature. At $200^{\circ} \mathrm{C}$ the decrease in methane flux is less than a factor of 2 . The $n$-butane flux is not influenced in the presence of methane. The permselectivities and separation results are summarized in Table 2. The permselectivity is completely different from the separation factor. These results can be explained by differences in sorption and diffusion behaviour of $n$-butane and methane. In $n$-butane/methane mixtures the $n$ butane adsorbs much more than methane at low temperatures [13]. The amount of $n$-butane molecules in

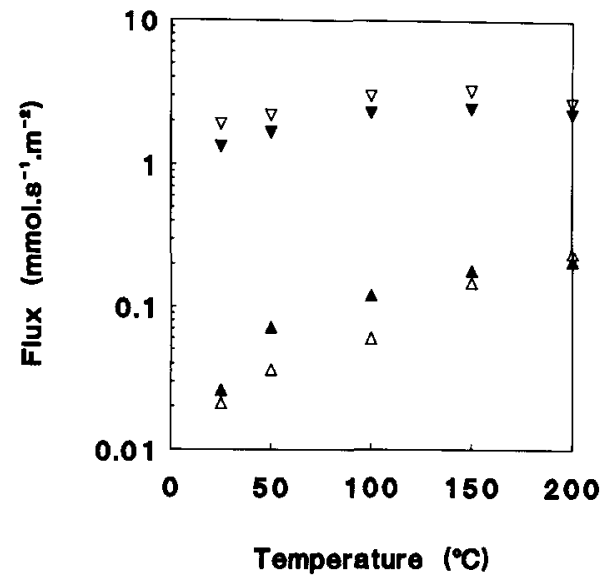

Fig. 11. Flux of $50 \mathrm{kPa} n$-butane $(\nabla)$ and $50 \mathrm{kPa}$ isobutane $(\boldsymbol{\square})$ as a function of temperature: $(\nabla, \boldsymbol{\square})$ single gas; $(\boldsymbol{\nabla}, \boldsymbol{\Lambda})$ mixture. the zeolite pore is much larger than the amount of methane molecules shown (Fig. 10a). The molecules cannot pass each other in the zeolite pore, so the $n$ butane molecules block the zeolite pores for the faster moving methane molecules. Separation is mainly determined by differences of both molecules in entering the zeolite pores (sorption). The methane diffusion constant is determined by the $n$-butane diffusion constant and the average distance between two sorbed ( $n$ butane) molecules. The increase in the methane flux with increasing temperature is caused by two effects: (1) increase of the $n$-butane diffusion constant, (2) increase of the average distance between two $n$-butane molecules in the pore. At high temperatures the amount of sorbed molecules in the zeolite pores is smaller (see Fig. 10b). In that case methane and $n$-butane can diffuse almost independently. Separation is based on sorption and diffusion and the permselectivity $\left(P_{\alpha}\right)$ and the separation factor $(\alpha)$ are almost equal.

Fig. 11 shows the separation of $n$-butane/isobutane as a function of the temperature. The separation of $n$ butane/isobutane decreases from $50\left(25^{\circ} \mathrm{C}\right)$ to 11 $\left(200^{\circ} \mathrm{C}\right)$. The $n$-butane flux is decreased by only a factor of 1.5 at $25^{\circ} \mathrm{C}$. The isobutane flux is the same as in the single gas flux. The difference in the permselectivity and the separation factor are not so pronounced for the $n$-butane/isobutane mixture compared to the $n$ butane/methane mixture.

The separation of $n$-hexane/2,2-dimethylbutane is 600 and $>2000$ at $25^{\circ} \mathrm{C}$ and $200^{\circ} \mathrm{C}$, respectively. The branched molecule, 2,2-dimethylbutane, can hardly 
enter the zeolite pore due to its large size. This high selectivity is a strong evidence that the membrane shows selectivity by size-exclusion and that transport in pores larger than the zeolite MFI pores (possible defects, etc) can be neglected, so a thin defect free zeolite MFI membrane has been prepared.

\section{Conclusions}

A thin polycrystalline $(<5 \mu \mathrm{m})$ defect free zeolite MFI membrane has been prepared. The variation in the flux of $n$-hydrocarbons in zeolite membranes is much smaller than expected from the difference in diffusion constants only. The flux is a function of the sorption and diffusion. In mixtures of strongly and weakly adsorbing gases the gas flux of the weakly adsorbing gas is suppressed by the sorption and the mobility of the strongly adsorbing molecule resulting in poreblocking effects. This also causes large differences in permselectivities and separation factors. High selectivities for the isomers of butane and hexane (respectively larger then $>10$ and $>600$ ) are a strong evidence that the membrane shows selectivity by size-exclusion and that transport in pores larger than the zeolite MFI pores (possible defects, etc) can be neglected.

\section{Acknowledgements}

Special thanks to P. IJben for performing the permeation measurements.

\section{References}

(1) A.F.M. Leenaars and A.J. Burggraaf, The preparation and characterization of alumina membrane with ultra-fine pores. Part 2 The formation of supported membranes, J. Colloid Interf. Sci., 105 (1985) 27.

|2| R.R. Bhave, Inorganic Membranes: Synthesis, Characteristics and Applications, Van Nostrand Reinhold, New York, 1991.
[3] M.D. Jia, K.V. Peineman and R.-D. Behling, Ceramic zeolite composite membranes. Preparation, characterization and gas permeation, J. Membrane Sci., 82 ( 1993) 15.

[4] W.J.W. Bakker, G. Zheng, F. Kapteijn, M. Makkee, J.A. Moulijn, E.R. Geus and H. van Bekkum, Single and multicomponent transport through metal-supported MFI zeolite membranes, in Precision Process Technology, Perspectives for Pollution Prevention, M.P.C. Weijnen and A.A.H. Drinkenburg (Eds.), Kluwer, Dordrecht, The Netherlands, 1993, p. 425.

[5] E.R. Geus, M.J. den Exter and H. van Bekkum, Synthesis and characterization of zeolite (MFI) membranes on porous ceramic supports, J. Chem. Soc. Faraday Trans., 88 (1992) 3101 .

[6] E.R. Geus, H. van Bekkum, W.J.W. Bakker and J.A. Moulijn, High-temperature stainless steel supported zeolite (MFI) membranes: preparation, module construction, and permeation experiments, Microporous Mater., 1 (1993) 131.

[7] J.G. Tsikoyiannis and W.O. Haag, Synthesis and characterization of a pure zeolitic membrane, Zeolites, 12 (1991) 126.

[8] W.M. Meier and D.H. Olson, Atlas of Zeolite Structures, 3rd ed., Butterworth-Heinemann, 1992.

19] D.W. Breck, Zeolite molecular sieves: Structure, chemistry and use, Wiley, New York, 1973.

[ 10] D.T. Hayhurst and A.R. Paravar, Diffusion of $C_{1}$ to $C_{5}$ normal paraffins in silicalite, Zeolites, 8 (1988) 27.

[11] D.B. Shah, S. Chokchal-acha and D.T. Haydrust, Measurements of transport rates of $\mathrm{C}_{4}$ hydrocarbons across a single crystal silicalite membrane, J. Chem. Soc. Faraday Trans., 89 (1993) 3161.

[12] Z.A.E.P. Vroon, K. Keizer, H. Verweij and A.J. Burggraaf, Transport studies of thin ceramic supported zeolite (MFI) membranes, to be published.

[13] Z.A.E.P. Vroon, Synthesis and Transport Studies of Thin Ceramic Supported Zeolite (MFI) Membranes, Ph.D. Thesis, University Twente, 1995.

[14] H.W. Deckman, A.J. Jacobson, A.J. McHenry, K. Keizer, A.J. Burggraaf, Z. Vroon, L.R. Czarnetzki, A.J. Bons, J.P. Verduijn and W. Mortier and E.W. Corcoran, Molecular Sieve Layers and Processes for their Manufacture, WO94/25151, 1994.

[15] E.R. Geus, Preparation and Characterization of Composite Inorganic Zeolite Membranes With Molecular Sieve Properties, Ph.D. Thesis, Technical University of Delft, Delft, 1993, Chap. 6.

[16] M.B. Rao and S. Sircar, Nano-porous carbon membranes for separation of gas mixtures by selective surface flow, J. Membrane Sci., 85 (1993) 253. 